\title{
Bendita tesis: tribulaciones personales e institucionales
}

\author{
Cristina Ambrosini ${ }^{1}$ \\ Andrés Mombrú Ruggiero² \\ El contexto de los estudios de posgrado
}

Hacia la mitad del siglo XX, no era poco frecuente que alguien se presentara socialmente con una tarjeta en la que se podía leer: "Fulano de Tal, Bachiller Nacional"., o "Mengano de Cual, Perito Mercantil". La obtención de un título secundario era motivo de prestigio y reconocimiento, daba cuenta de que se trataba de una persona con "estudio", merecedora de mejores oportunidades en la vida. Algunos alcanzaban títulos universitarios: ingenieros, arquitectos, médicos, abogados. Como ocurre todavía hoy, estos últimos ostentando un título de doctor sin serlo. La denominación obtenida en forma consuetudinaria se remonta al principio Ilustrado para el cual alguien que conoce en profundidad un tema se manifiesta en carácter de docto; en estos casos, doctos en la ley y doctos en temas de salud. De cualquier manera hasta bien entrado el siglo XX los sectores que tenían acceso a la educación en general y a la educación superior eran muy restringidos. Por otra parte, aquellos saberes a los que se accedía eran preciados porque permitían el acceso a cierto estatus, pero además eran duraderos.

Aquellos conocimientos y destrezas que se adquirían duraban para toda la vida, e

1Cristina Ambrosini es Doctora en Filosofía por la UBA. Profesora regular del CBC de la UBA en la materia Introducción al pensamiento científico. Profesora regular de la UNLa en el área de ética, coordinadora académica de la Maestría en metodología de la Investigación Científica de la UNLa, dicta materias de posgrado. Autora de Del monstruo al estratega, Ética y juegos, Editorial Educando 2007, coautora con Rubén Padlubne de Ficciones posibles. Saberes filosóficos, semiológicos y científicos a través de la literatura, Biblos, 2014 y coautora con Carlos Asti Vera de Estructuras y procesos. Temas de Epistemología, Educando (2005) y de Argumentos y teorías. Aproximación a la epistemología, Educando (2009)

2 Andrés Mombrú Ruggiero es Dr. en Filosofía por la UNLa, Mg. y Especialista en Metodología de la Investigación Científica por la UNLa, Lic. En Filosofía por USAL, decente regular Asociado de la materia Metodología de las Ciencias Sociales, CBC, UBA; Titular de IPC UNLa, Profesor regular en Lógica Aplicada a la Investigación y Seminario de Redacción de Tesis y Coordinador Pedagógico en la Maestría en Metodología de la Investigación Científica en UNLa. Ha publicado numerosos libros y artículos en revistas de su especialidad, entre ellos: "Encrucijadas del Pensamiento, Análisis críticos del quehacer científico", con Sonia Durand, editorial Gran Aldea, 2004; "Filía (Filía) Uno de los posibles recorridos a través de la Filosofía de Occidente, con Carlos Rodríguez Murúa, Ed. Autor, 2011; Paralaje: Los giros y las controversias en el campo de la epistemología y sus consecuencias metodológicas en la investigación científica. Ediciones L.J.C. 2012; El Hacedor de Tesis en colaboración con Alejandro Marjetic, L.J.C. Ediciones, (reedición) 2013. 
incluso para la vida de la generación siguiente, por ello es que tanto en los oficios, como en las profesiones y en las disciplinas científicas, la trasmisión de los conocimientos era restringida dentro de las familias, del gremio o de la disciplina, seguramente como una herencia de la circulación del conocimiento en la Edad Media. Pero en la medida en que los conocimientos se incrementaban y al mismo tiempo decaía su valor, al ser sustituidos por otros, la lógica de su producción, circulación y uso se modificó dramáticamente. El mismo saber, convertido en mercancía respondía a la dinámica del vértigo de los mercados, era necesario sacarle el máximo provecho en el menor tiempo posible antes de que caducaran. Los imperativos de rápida obsolescencia que comenzaron a imponerse en los campos de la moda y la tecnología también se impusieron en la sociedad del conocimiento, la cual ve desbordada su capacidad crítica a causa del tsunami que produce la sociedad de la información. Más allá del hecho mismo de la información, del conocimiento, de los saberes y de las capacidades como mercancías, se encuentra, como en otros ámbitos de la producción el problema de la contaminación.

El viejo dicho, "el saber no ocupa lugar" es falso, y no nos referimos a las progresivamente arcaicas bibliotecas, ni a los a los crecientes terabyte, petabyte, exabyte, etc., conformadores de laberintos insondables de información, sino a la dificultad para diferenciar los conocimientos y saberes "significativos" para la vida de las personas de aquellos cuyo fin es el sostenimiento de las lógicas productivas a cualquier costo. Los conocimientos y saberes pertinentes para la realización de determinados proyectos personales y colectivos se ven "contaminados" por una enorme cantidad de requisitos externos, enajenantes, parasitarios de los primeros, que terminan consumiendo buena parte de la energía que debería estar destinada a los primeros. El mito de que el conocimiento es bueno por sí mismo ha impedido discriminar la finalidad a la que apunta. Disponer de recursos para algunas áreas y abandonar otras supone una decisión que facilitará ciertos desarrollos e impedirá otros. La decisión sobre que saberes fomentar y promover implica no sólo una decisión epistemológica, sino también política y ética. No sólo es importante saber, sino además saber qué se sabe y que consecuencias tienen esos saberes y su implementación sobre nuestras vidas. Lamentablemente el conocimiento que se promueve compulsivamente en la sociedad del conocimiento no apunta a la "sabiduría", ni siquiera como han supuesto muchos a partir de las tradiciones aristotélicas a la "felicidad", o más discretamente a la "realización" personal y social, sino que en la mayoría de los casos sigue apuntando a la supervivencia de muchos, mediante producciones genuinas o parasitarias y a la acumulación inaudita de riquezas por parte de unos pocos.

\section{Nuestro contexto general}

¿Qué ha impulsado a aquel Mengano o Perengano, bachiller o perito mercantil a tener un título universitario? ¿Qué ha motivado a aquel que ha obtenido un título de grado a obtener títulos de posgrado que van desde cursos de capacitación, pasando por especializaciones, maestrías, doctorados, hasta posdoctorados? Hoy causaría gracia cualquiera que se presente con una tarjeta que lo acredita como bachiller o perito mercantil, pero también, en situación similar, han caído los títulos de grado e incluso los de posgrado, ya que además esos títulos tienen que estar respaldados con producciones, publicaciones, asistencias a congresos, todos matemáticamente tabulados para la meritocracia que asigna reconocimientos y recursos. Se podría decir que el vértigo y la rápida obsolescencia de los 
conocimientos obliga a una formación permanente, pero el alto índice de fracaso y deserción muestran que la capacitación no pone en sintonía esfuerzo, recursos y resultados.

Alguien dijo sobre la tecnología que: "La tecnología viene a resolver una cantidad de problemas que antes del desarrollo tecnológico no teníamos”, se podría argumentar en contra que también ha resuelto problemas que sí teníamos y que los ha resuelto para muchos, pero lo que queda en entredicho es $L A$ TSSS el balance de problemas que ha resuelto en comparación con los que ha suscitado. Algo similar parece ocurrir con la investigación NO SE CREA científica que emana de la educación superior. Lo que se presenta como un hecho es que la posibilidad de mejoramiento (por lo NI SE DESTRUYE menos a mediano plazo) de las sociedades menos desarrolladas es apostar a la educación para la investigación y el desarrollo. $S O J O S E$ Lo que significa para esas sociedades un esfuerzo mucho mayor que para las del primer mundo. Numerosos estudios de CEPAL POSPONE ponen de manifiesto que en las últimas dos décadas la inversión en educación superior en América Latina ha producido un significativo incremento de esfuerzo de las personas y las familias tanto como del sector público y del privado en educación superior. El crecimiento de la matrícula ha crecido de 7 millones a 28 millones en las últimas dos décadas. Lo que ha cambiado entre la década de los 90 y la primera década del siglo XXI es el modelo político que impulsa las políticas en educación superior.

El neoliberalismo de los 90 apuntaba a una internacionalización y privatización de los servicios públicos, entre ellos la educación, en tanto que en la última década los proyectos integradores e inclusivistas de América Latina han dado mayor apoyo a las instituciones públicas y a la visión de la educación superior como un bien público social. De cualquier manera, según los especialistas, el promedio de inversión para la región es de aproximadamente el 1,5\% del PBI, lo cual resultaría insuficiente para atender a todas las demandas educativas y principalmente para sostener proyectos de investigación relevantes y sustentables. A esto debe sumarse otro dato importante. Según IESALC CINDA, 2005, ( Instituto para la Educación Superior de América Latina y el Caribe), las Ciencias Sociales, Empresariales y Jurídicas se llevan el $42 \%$ de la matrícula, en tanto que las ingenierías, industria y construcción el 14\%, educación y salud rondan el 10 $\%$ respectivamente, humanidades y arte el $6 \%$, otros el $2 \%$ y la agrícola (¿?) el $2 \%$. Se puede ver que sectores relevantes como el vinculado con el agro, por ejemplo, tienen una matrícula muy baja, para un sector productivo tan relevante, en tanto que las administrativas, de corte más burocrático son las de mayor matrícula y por lo tanto las que más recursos insumen. Estas deficiencias también se expresan en el muy bajo índice de publicaciones científicas, aproximadamente un 2,6\% de toda América Latina contra un $42 \%$ de Europa y un 31,8 de EE.UU.

Ahora bien, de los que realizan estudios de posgrado en América Latina y también en Argentina, sólo un $10 \%$ alcanza el título. ¿Quiere esto decir que se ha perdido un $90 \%$ de esos recursos, tanto por parte de los cursantes como por parte de las instituciones? Buena parte de las deserciones de ese $90 \%$ se dan muy cerca de haber finalizado los estudios, o incluso de haber concluido con ellos. Sin el título que se obtiene a partir de haber aprobado la tesis el reconocimiento institucional no se alcanza. Sin embargo, esto 
no quiere decir que los conocimientos y las capacidades adquiridas no se pongan en juego en los desempeños profesionales. Las mismas instituciones se benefician de disponer de personas capacitadas y formadas pero que no pueden acreditar esos conocimientos, eso se traduce en salarios inferiores para desempeños superiores. Esta situación surge de la siguiente ecuación: hay carreras largas (o que se hacen largas por distintos motivos), tanto en grado como en posgrado; para el ejercicio profesional de ciertas disciplinas no se requiere título o matrícula, la obtención del título se ve como lejana o inalcanzable, pero el ejercicio profesional es inmediato aunque se gane menos (o igual) que como titulado. Conclusión es más "negocio" trabajar aunque se gane menos que realizar el costoso esfuerzo de titularse. Para otros, como vimos, la titulación es imprescindible para la obtención de mejoras laborales. La realidad de los profesionales es que buena parte de las motivaciones para realizar posgrados no radican en el amor por el saber, sino en la expectativa, en algunos casos de mejorar los ingresos, en muchos, apenas en conservar el trabajo frente a la creciente competencia. Otro aspecto a tener en cuenta es que la misma práctica disciplinar obliga a una formación constante que se da mediante la práctica y los conocimientos que espontáneamente se adquieren a través de lecturas, cursos, inter-capacitación laboral. ¿Qué se busca en estos casos con la obtención de un título de posgrado? En ocasiones validar conocimientos que ya se poseen.

En los países centrales la situación parece ser la inversa en cuanto al promedio de

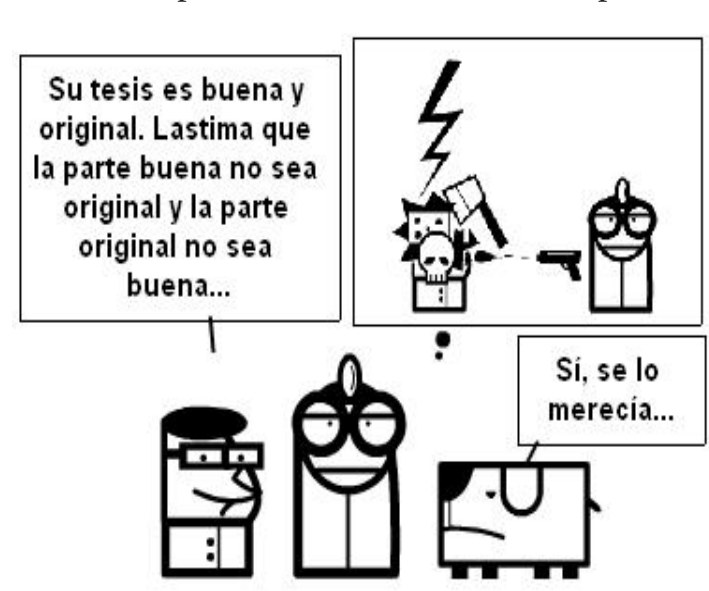
graduados, va del $50 \%$ al $90 \%$ de titulaciones. ¿Qué similitudes y diferencias encontramos entre estas dos realidades? En relación a los estudiantes no se puede dejar de tener en cuenta su condición sociocultural. En argentina el $90 \%$ de los estudiantes de posgrado estudian y trabajan y se encuentran motivados por expectativas económicas, esto no es ni bueno ni malo en sí mismo, pero determina que si los obstáculos o los resultados no satisfacen esas expectativas entonces es posible una mayor tasa de deserción, a diferencia que cuando las motivaciones son otras como por ejemplo la vocación. En nuestro país la edad promedio es entre los 25 y 30 años, pero se advierte un alto índice de quienes superan de 35 años. La mayoría de los estudios de posgrado son pagos. En los ámbitos privados son costosos, aunque en los públicos son mucho más accesibles. Para la gran mayoría de los cursantes los sistemas de becas consisten en restricciones o no pago de la matrícula y la cuota, esto sobre todo para los estudiantes que forman parte de una institución superior, son pocos los casos en que los estudiantes cobran becas lo suficientemente importantes como para realizar sus estudios sin trabajar.

En EEUU y Europa los estudios de posgrado, principalmente las maestrías se realizan a continuación de los estudios de grado. Los estudiantes prolongan su estatus universitario y su dependencia familiar. Esto también es posible por la duración de los posgrados, muchos de ellos menores de un año contra los dos o tres años que duran en la Argentina. 
El organismo principal para establecer la calidad educativa en Argentina es la CONEAU, (Comisión Nacional de Evaluación y Acreditación Universitaria). En la Resolución Ministerial 160/11 se establece la diferencia entre especializaciones, maestrías y doctorados. La especialización apunta a una profundización de los dominios en los desempeños profesionales. La maestría lo mismo pero con alcance profesional y/o académico. Mientras que la orientación profesional apunta a profundizar prácticas y saberes profesionales, la académica apunta a la formación para la investigación científica y los procedimientos metodológicos, en definitiva para la apropiación de herramientas para la producción de conocimiento en áreas disciplinares. En tanto que el doctorado exige la producción de aportes originales en un área específica. Estas definiciones nos ponen frente a un viejo problema que surge en la misma formación de grado, ¿una educación universitaria con un perfil profesional o académico? ¿ Una preparación para la práctica profesional o para la investigación científica? De hecho los estudiantes de grado transitan casi en forma conjunta, en los mismos claustros los dos tipos de orientaciones. No está mal que esto ocurra, pues la formación que excluye a estos grupos, como por ejemplo se practicó en algunas universidades de la URSS, desvincula la producción de conocimiento científico de las prácticas y los problemas concretos del resto de la sociedad. Esta situación hace que, en parte, se corra el peligro de no conseguir un perfil nítido para el cumplimiento de los propósitos de esas orientaciones.

Entendemos que en las carreras de corte profesional la formación se encuentra orientada principalmente para la práctica o desempeńo profesional, en tanto que, en las de corte académico el énfasis está puesto principalmente en la investigación. Ello implica modos de estudio, enfoques de problemas, didácticas diferentes. Las especializaciones no presentarían mayores dificultades cuando están llevadas adelante en sus áreas disciplinares específicas, aunque suelen producirse enfoques demasiado estrechos que no son capaces de revisarse a sí mismos a la luz de otras perspectivas epistemológicas y metodológicas. Por ello la importancia del desarrollo de la investigación interdisciplinaria incluso en sus pareas de formación. Pero, ¿qué pasa con las maestrías cuando los alcances profesionales y/o académicos toman más partido por la conjunción que por la disyunción? ¿Cómo se conjugan desde una perspectiva pedagógica y disciplinar estos dos universos? Se trata sin dudas de una situación conflictiva, pero de una conflictividad que merece ser encarada, ya que de su conjunción surge una dimensión relevante que aporta un plus valor a la formación de posgrado. En la actualidad un profesional no puede ser un mero aplicador de técnicas, para eso, cada vez más y de modo más eficiente están las máquinas y las computadoras. ¿Cuál es entonces la tarea más significativa de un profesional? Tomar decisiones. Por supuesto que tiene que tener un conocimiento técnico exhaustivo, pero su capacidad principal se manifiesta en la toma de decisiones, no sólo con respecto a esas técnicas, sino además en relación con ese universo cambiante que es su producción teórica disciplinar. Un profesional no está obligado a producir conocimientos como un científico, pero tiene la obligación de poder interpretar adecuadamente la producción teórica de su área. Un contador no es una calculadora, ni tampoco un creador de teorías económicas, pero debe poseer la capacidad para reflexionar sobre los que los cientistas sociales de su disciplina producen. Del mismo modo un científico debe estar al tanto de los diversos problemas que se producen en su campo a raíz de sus desarrollos teóricos. Entendemos que parte de los fracasos y la deserción en muchos posgrados es a causa de las dificultades para ensamblar armoniosamente estos dos universos. 


\section{Nuestro contexto particular}

El campo académico tampoco es homogéneo. Los modos de investigar y de comunicar los resultados de las investigaciones difieren, no solamente entre las ciencias naturales y sociales, sino también entre disciplinas próximas. Si bien la praxis científica implica

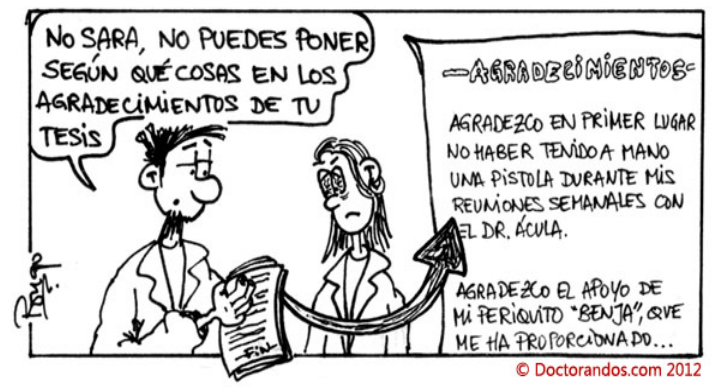
una dialéctica entre teoría y empiria, los sesgos teóricos y empíricos imponen diferentes modalidades, que a la postre generan prácticas y discursividades no siempre afines. Esta situación es común entre físicos teóricos y físicos experimentales, pero también entre antropólogos teóricos y antropólogos de campo. Los ámbitos de producción discursiva también son heterogéneos entre disciplinas y dentro de las mismas disciplinas, en relación con las prácticas disciplinares. Quienes trabajan en equipos de investigación están acostumbrados a la elaboración de informes y de "papers" que surgen como recorte de una tarea colectiva, que en general es un proyecto de investigación del que participan. En el campo de la filosofía y de las artes la tarea suele ser más solitaria, dando lugar a ensayos y artículos. Estas últimas formas de escritura también son una modalidad de comunicación en el ámbito profesional, la dificultad que plantean es que en ocasiones su carácter especulativo, o su modalidad periodística, impide distinguir la mera opinión (doxa, pareceres infundados) de los juicios fundados (episteme, fundados no sólo empíricamente y argumentalmente, sino integrando perspectivas de las epistemologías ampliadas). Pero algo tienen en común las distintas disciplinas en su instancia formativa, principalmente en el grado, y es que la escritura se limita la mayoría de las veces a responder preguntas, como en los exámenes abiertos, o a someterse a la modalidad de múltiple choice, en el que la escritura se encuentra ausente. En contadas ocasiones se piden monografías y en ellas se suele evaluar sus contenidos conceptuales, pero no el cumplimiento con esa forma de comunicación que es la escritura académica. Como señala Roberto Follari:

Y nos queda, lastbutnotleast, la cuestión de la escritura. Hoy los estudiantes no saben escribir. Mucho se ha dicho al respecto, y sin duda que la condición de posmodernización cultural, con el auge del mundo visual, es responsable estructural de esta situación. También las fallas en la educación de nivel primario y medio, y la falta de suficiente entrenamiento durante las carreras universitarias de grado. Es más: los alumnos muestran a veces dificultades de comprensión lectora, hasta cerca del final de sus licenciaturas. Todo esto hace que la tarea del director, a menudo se asimile a la de un corrector de estilo dentro de una editorial: corregir acentos, mayúsculas, comas, signos, palabras y construcción gramatical de frases.(Follari, 2001)

Entendemos que la dificultad en la escritura es sólo parte de la dificultad de comunicación. No sólo no se sabe escribir, tampoco se sabe hablar. El idioma se ha empobrecido, el conjunto de palabras que se usan se ha ampliado por un lado pero se ha restringido notablemente por otro, siempre haciendo alusión a grupos que deben al habla y a la escritura 
parte de su razón de ser. Hay más palabras, pero más pobreza gramatical y en consecuencia menor capacidad significativa. La pobreza de la palabra se convierte en pobreza del espíritu.

La tesis es un tipo particular de escritura que se diferencia de la monografía, el ensayo, el artículo, el informe o la ponencia. En la vida académica y por ende en los estudios de posgrado se dan todos estos tipos de escritura, es por ello que es necesario distinguirlos y manejarlos. Sin embargo, como hemos visto, a lo largo de la carrera de grado es escasa, por no decir nula, la formación para estos menesteres, incluso hay muchas carreras a las que se accede al título de grado sin la presentación de una tesis. Luego, cuando llega la hora de realizar un trabajo de esta naturaleza, suele reinar el desconcierto y la angustia, a causa de la necesidad de encarar una tarea para la que no se está preparado. Es que una tesis implica un tipo de escritura esencialmente académica, esto es, reflexiva, crítica, argumentativa; supone una dialéctica entre la teoría y la empíria, el manejo de procedimientos metodológicos específicos y propios anclados en sus fundamentos epistemológicos. En definitiva, una tesis es una investigación, una tarea propia de investigadores. Es por ello que les cuesta menos a aquellos que tienen a la investigación como parte de su "metier" cotidiano, pero les cuesta mucho más a los que se han formado sólo para ejercer una profesión. Este problema se agrava cuando los directores de tesis pueden ser idóneos en su área disciplinar, pero carecen de los conocimientos sobre formas de investigación, o aunque sean investigadores no tengan la capacidad de acompañar a su dirigido en el proceso de elaboración de la tesis. Otro problema a tener en cuenta es la dificultad para identificar las herramientas metodológicas que se condicen con el objeto de investigación y las elecciones epistemológicas del marco teórico. El problema se presenta como menos grave para maestrías orientadas en forma selectiva sobre un área disciplinar, ya que las elecciones metodológicas suelen ser propias y pertinentes; se complica mucho más cuando los concurrentes provienen de diferentes disciplinas y no aparece claridad en la pertinencia de la elección metodológica y epistemológica. Sumado a todos estos problemas se encuentra el específico de la escritura de la tesis. Esto sin tener en cuenta los debates epistemológico-metodológicos que atraviesan a todas las disciplinas.

Hasta tal punto la elaboración y presentación de la tesis se ha convertido en un obstáculo que la psicóloga Venezolana y Doctora en Ciencias Elizabeth Valarino, ha establecido las categorías de síndrome de TMT (Todo menos Tesis) y de TMI (Todo menos Investigación), ellos se expresan como:

[...] el concurso de trastornos, impedimentos, obstáculos, actitudes, sentimientos y conductas inadecuadas, ausencia o deficiencia en algunas destrezas o lagunas de conocimientos, que presentan o enfrentan los estudiantes, profesores y profesionales, cuando abordan la tarea de diseñar, planificar, desarrollar, escribir, supervisar y publicar trabajos de investigación o proyectos a largo plazo, como pueden ser trabajos de grado, tesis, trabajos de ascenso, artículos científicos, monografías, informes técnicos, reportes o similares, así como en la tarea de enseñar sobre dichos procesos. (Valarino, 1997, p. 255)

Entendemos que los motivos psicológicos concurren con otros de carácter social, cultural, económico, e institucional. Una manera de aproximarse a esta problemática 


\section{2/ Perspectivas Metodológicas /19/Vol. II /Año 2017}

consiste en analizar las dificultades que se presentan a la hora de la elaboración de la tesis, de encarar el proceso de investigación ordenando cada una de sus etapas y de plasmar en un documento escrito con claridad argumentativa cada una de las partes que darán forma "física" a esa unidad que es la tesis, en la instancia de lo que se da en llamar Taller de Tesis, Taller de Escritura de Tesis, Seminario de Tesis y otras denominación.

\section{No es bueno que el tesista esté solo}

Nuestra experiencia como docentes de Seminarios de redacción de tesis y lectores de tesis ya sea para orientación de alumnos y colegas o como jurados indica que entre las principales dificultades y obstáculos para la presentación de tesis de posgrado se señalan la desorientación y el aislamiento. En contra de la creencia firme de que es la falta de tiempo el principal motivo que lleva al abandono de las tesis, en nuestra experiencia a partir del contacto con tesistas hemos advertido que la principal desmotivación está dada por el desánimo que produce la falta de "acompañamiento" en el difícil momento de la escritura. En nuestra tradición cultural se supone que un escritor es como un navegante solitario que necesita estar apartado del mundo para poder concretar su tarea sin distracciones mundanas y sin la contaminación de ideas ajenas que afectarían la originalidad. En parte es cierto para el caso de la creación literaria donde la metáfora de la concurrencia de las "musas" inspiradoras se asocia a la intimidad de una voz interior, pero en el caso de la escritura académica se requiere la colaboración de muchas personas entre las que el director de tesis es una pieza fundamental sin desmerecer el aporte de profesores, colegas, amigos y familiares que pacientemente "contengan" al autor de la tesis en momentos de crisis.

En el último Seminario de redacción de tesis dictado en la Maestría en Metodología de la Investigación Científica de la UNLa, como parte del Examen final se administró un Cuestionario donde la primera pregunta fue ¿Por qué cree que no se termina una tesis? Recabaremos a modo de ejemplo algunas respuestas.

\section{Respuesta 1}

Coincido con Arnoux y otros (2004) que una tesis demanda "una extensión, un nivel de conceptualización original, un grado superior de integración de conocimiento y una capacidad de autoorganización y regulación del trabajo autónomo que solo pueden vehiculizarse por medio de un dominio escritor difícilmente obtenido en etapas anteriores". Habitualmente estas competencias necesarias para completar una tesis no se adecuan con la "ecuación personal", descripta por Mombrú (2013) es decir aquellas exigencias de tipo laboral y/o personal. Por eso, que se hace necesario el acompañamiento del director de la tesis y la interacción cooperativa con otros maestrandos.

\section{Respuesta 2}

Creo que un proceso de elaboración de tesis se ve atravesado por diferentes factores, por un lado los emocionales/personales, y por otro, los profesionales/disciplinares. Hay componentes que afectan a todo tesista emocionalmente, en tanto la dificultad que está asociada socialmente a la elaboración de una tesis y a la graduación en si a partir de la misma. Además, el tesista que se encuentra en ese proceso y enfrenta este desafío, está terminando un recorrido 
que amerita balances y cuestionamientos, saca a relucir inseguridades respecto de la manifestación pública (a sus pares) de sus propuestas y concepciones. Esto, sumado a los complejos tiempos personales y laborales de la actualidad, que impiden dedicarse exclusivamente a la realización de un estudio en la mayoría de los casos, complejizan la terminación de una tesis.

\section{Respuesta 3}

Considero que existen varias razones por las cuales no se termina una tesis. Una de ellas es el estado de aislamiento, de soledad, del tesista que no encuentra acompañamiento (tutorías o seminarios) ni instancias de dialogo e intercambio para llevar adelante su tarea. Otra razón es la falta de experiencia en la producción de este tipo de escrito puesto que, su gran mayoría, nunca ha leido, por ejemplo, una tesis. Este se relaciona con la ausencia de prácticas comunicativas especificas. También se puede mencionar que otra razón es la falta de tiempo que se relaciona con la ausencia de un cronograma tentativo y un plan personal donde se combinen los pasos y nuestro tiempo real dedicado a la producción de la tesis. La falta de tiempo y una mala organización personal del mismo provocan una discontinuidad de la tarea. Otra razón es el tiempo que el director de la tesis le dedica a nuestro trabajo y, a veces, la falta o ausencia de encuentros personales dedicados entre tesista y director para analizar el avance de nuestra producción.

\section{Respuesta 4}

Es sabido que la brecha entre la finalización de la cursada de los estudios de posgrado y la presentación de las tesis en muy grande. Considero que las causas son múltiples. Entre ella la presentación de la tesis es una trayectoria más solitaria que involucra autodisciplina por parte del alumno. Otro aspecto a considerar es la brecha entre la teoría y la práctica, la tesis implica llevar adelante un proyecto, responder a la buisqueda de conocimientos en relación a un problema real. Los tiempos concretos no son un aspecto menor los estudios de posgrado por lo general son realizados por personas dividen sus tiempos entre el trabajo, estudio, vida familiar y personal y el caso de las mujeres hay que considerar la crianza de los niños en las etapas de adultez temprana.

\section{Respuesta 5}

Creo que existen dos dificultades importantes a la hora de terminar una tesis, la primera es la falta de tiempo en exclusividad para dedicarse a ella, no existen becas accesibles para ello, y las múltiples actividades conspiran contra la finalización, la segunda es la falta de acompañamiento, la soledad, por mejor que sea un director de tesis, el problema principal es la falta de instancias intermedias durante la realización de la tesis, como talleres de apoyo entre alumnos, casi a modo de autoayuda, o instancias de encuentro con docentes de la maestría, etc., que permitan revitalizar o renovar las ganas de seguir incluso desde el lugar de ir (al lugar fisico) de la facultad.

\section{Respuesta 6}

Son varios los factores que imposibilitan la terminación de una tesis. Los mismos están vinculados de manera directa o indirecta con lo que particularmente denomino "Condiciones de posibilidad para "proveniente del sector público o privado, empresa o ámbito académico, ciencias biológicas o sociales, desarrollo del hábito de la escritura, etc., y del peso relativo de cada 
uno en el contexto de cada investigador. Por ejemplo no es lo mismo una persona que proviene de una universidad con experiencia en presentación de trabajos, que de la actividad profesional, ya que el primera está más favorecido por el entorno académico que el segundo. Otro de los condicionamientos para terminar la tesis se podría relacionar con recursos económicos, financieros e infraestructura (por ej. Laboratorios) identificado con la disciplina cientifica a la que pertenece el investigador. Es obvio que las provenientes de las ciencias biológicas son dependientes de estos recursos. En el pais existe una desigual distribución de estos recursos (hay excepciones) ya que coexisten universidades más provistas que otras de equipos, infraestructura y de cientificos o investigadores formados, con el tiempo suficiente para acompañar o dirigir tesis de post grado. Desde el punto de vista individual, creo que el tiempo es otro factor que influye dado que existe la necesidad de cumplir, primero, con las obligaciones laborales si uno no es investigador de carrera, cuyo ingreso es exclusivamente es producto exclusivamente de su actividad profesional y en segundo lugar si queda tiempo, dedicarse a la actividad de investigación.

En resumen, si se generara un ámbito más accesible para la investigación, especialmente para los tesistas de maestrías y doctorados, que implicara acompañamiento y que diera la oportunidad de consulta permanente, más las condiciones de infraestructura adecuadas (en función del tipo de tesis y que es factible de lograr con politicas explícitas orientadas a facilitar el trabajo de los mismos) se podria incentivar la conclusión de los trabajos de tesis en todo el territorio del país. No obstante reconociendo aún que desde el gobierno nacional se ha encarado una politica de fuerte apoyo a la investigación e innovación, es evidente que las mismas no hacen hincapié en esta problemática.

\section{Respuesta 7}

Para responder esta pregunta tendré en cuenta las experiencias vividas al compartir espacios con colegas de mi disciplina, son muchos los que han terminado el cursado de diferentes maestrias y la mayoría no han entregado la tesis, los problemas que se plantean son: falta de tiempo para poder dar continuidad al trabajo de investigación, sobre todo en la etapa de elaboración del informe; escasos recursos gramaticales que impiden una buena redacción y también un no reconocimiento de los errores cuando son marcados por el Director de Tesis o los profesores en las entregas de informes de avance he escuchado expresiones como "no lo entiendo y no me entiende, no sé qué quiere que escriba"; falta de incentivo, ya que en general, el terminar la tesis no le significará ningún cambio en relación a su jerarquia laboral; y sentimientos de que se está investigando algo que no tiene mucha importancia o que un enfermero no tiene capacidad para un trabajo tan profundo.

\section{Respuesta 8}

Creo que una mala planificación y la gran cantidad de horas que requiere la realización de una tesis son las principales trabas. Al planificar mal y al no cumplir con el cronograma inicial que el maestrando realiza, se genera una pérdida de interés a realizar tanto esfuerzo junto con la falta de obligatoriedad de presentar informes preliminares hacen que los tiempos se hagan demasiados largos perdiendo el hilo del proyecto.

\section{Respuesta 9}

Si bien es difícil generalizar al respecto, me parece que la problemática remite a dos cuestiones, el pluriempleo de los tesistas que demanda de ellos un esfuerzo desmedido y creo por 
otra parte que la tesis se debe empezar a escribir cuando uno está cursando y no cerrando la carrera; dejando al alumno en un umbral donde lo que queda a veces es soledad pura.

\section{Respuesta 10}

Entiendo que la problemática para la elaboración de un trabajo de tesis no tiene que ver con un factor específico, sino que más bien estamos ante la presencia de una problemática multifactorial. De todas maneras, desde mi óptica, el tiempo del que uno dispone asoma como el principal obstáculo (en términos de lo cotidiano y no del tiempo administrativo de entrega del que uno dispone). Considero que en gran porcentaje todos los que tienen la posibilidad de presentar un trabajo de tesis, tienen el bagaje académico suficiente para su elaboración. De todos modos es aqui donde las complicaciones laborales, familiares, etc., abortan la misión de finalizar el trabajo.

Otra problemática de mayor peso, refiere a la conjunción de la falta de entrenamiento del tesista para su elaboración, sumado a todas las dificultades con las que uno puede encontrarse cuando intenta plasmar una idea en un papel (inconvenientes para encontrar su propia voz). En esta instancia los directores de tesis, los cuales pueden cooperar para salir del "bache" en el cual uno se encuentra inmerso, en muchas ocasiones disponen aún de menos tiempo que el tesista, generando una interrupción en el flujo de comunicación entre ambos.

\section{Respuesta 11}

Uno de los inconvenientes surgen en la enseñanza en el pregrado: históricamente, en gran número de profesiones a lo largo del proceso formativo, se elimina la curiosidad y la duda sobre los saberes previos. El aprendizaje está basado en la repetición y el entrenamiento más que en la reflexión y en su reemplazo se apuntalan actitudes acriticas y conformistas, un no cuestionamiento a lo establecido. Al mismo tiempo generalmente se realizan análisis superficiales acerca de los procesos involucrados en la producción de conocimientos y las condiciones de su realización. Sumándole, ya en la instancia del trabajo final, un escaso o nulo conocimiento previo del tema propuesto para investigar.

En los posgrados encontramos, en gran parte, estudiantes con experiencia laboral en áreas en donde prevalecen los aspectos técnico y procedimentales, probablemente ninguna o escasa participación en equipos de investigación y un reducido número que acredita realizaciones por lo menos cercanas a lo cientifico. En forma conjunta, se identifican las limitaciones del tiempo disponible versus las expectativas en función de la tesis, y las posibilidades personales, profesionales y laborales. Esta situación es percibida inicialmente por el alumnado con temor por un lado (sustentado por el imaginario construido en torno a la investigación como actividad de "elegidos") y curiosidad. El reto es vencer el primero y aprovechar lo segundo en pos de producir una motivación tal que se mantenga a lo largo del proceso investigativo.

\section{Respuesta 12}

En mi experiencia personal he realizado una investigación sobre dificultades percibidas para la elaboración de la tesis en estudiantes de grado de la carrera de psicología de una Universidad privada y he observado que no se diferencian demasiado de lo que ocurre en posgrado. Entre los obstáculos percibidos, los más destacados eran: a) falta de formación en 
116/ Perspectivas Metodológicas /19/Vol. II / Año 2017

investigación, b) temor a no poder producir nuevos aportes, c) temor al manejo de la teoría, d) dificultades en la escritura y falta de acompañamiento del director.

Volviendo a las carreras de posgrado y buscando priorizar el factor de mayor peso, considero que en la formación universitaria no se transmite una "cultura de la investigación" siendo la consecuencia más inmediata de este déficit, la falta de realización de tesis de posgrado

\section{Respuesta 13}

De acuerdo a los conocimientos adquiridos en el módulo seminario de tesis, se pueden advertir problemáticas y dificultades que se presentan durante todo el proceso de desarrollo del plan de tesis, entre ellas se encuentran:

-Un intercambio de dialogo con el director poco fluido, que impide llevar adelante lo pasos del plan. Es común que los tesistas presenten un diálogo pobre con sus directores, aun cuando lo tienen y valoran, lo consideran insuficiente. Consideran que hay preguntas que no pueden plantearle al director, ya que estiman que solo deben hacerle consultas pertinentes a la investigación.

- Muchas veces el tesista no da a leer los avances al Director, y continua trabajando solitariamente, construyendo un argumento que aún no han sido revisados, por colegas, o personas pertinentes al tema en cuestión o que sepan de gramática, esto hace que los tiempos se extiendan cada vez más.

- Un inadecuado manejo del aparato crítico: suele advertirse en muchos trabajos una falta de criterios para expresar con cierta claridad, los objetivos y los puntos de anclaje de la investigación, diagnósticos que no están bien definidos.

- Falta de entrenamiento: es común que el alumno que inicia por primera vez un plan de tesis no posea una conceptualización sobre un discurso, ni sobre la escritura como práctica comunicativa especifica, ni sobre el campo académico pertinente en cuestión. También presentan un escaso entrenamiento en la escritura de textos académicos, por lo que se trata de un alumno que además de desconocer el producto que tiene que resolver, tampoco conoce las características del proceso que debe !levar a cabo para concretarlo.

- Dificultades en la conceptualización sobre el discurso, que obstaculiza la toma de decisiones adecuadas a la hora de redactar.

-Autonomia: la libertad que uno tiene para elaborar el plan, muchas veces perjudica la evolución de la misma, la autonomía juega en contra de la producción final. El investigador deja pasar lo tiempos pautados en la investigación, (lo dejo para más adelante, o lo termino la próxima semana o luego lo termino), obligando constantemente a replanificar los tiempos estimados de cada etapa.

\section{Algunas consideraciones como conclusión}

La "ecuación personal" a la que se hace referencia en una de las respuestas, representa no una situación individual aislada, sino el común denominador que más allá de las 
particularidades impone el contexto social, económico, laboral, familiar e institucional para el área de la UNLa, pero que entendemos que también tiene aspectos comunes con el resto del área latinoamericana. Hemos visto que el tiempo que se pueda dedicar a la producción de la tesis, en medio de exigencias laborales, familiares, institucionales no es el elemento que más conspira contra esa realización. Aunque no deja de ser importante se puede apreciar que afecta del mismo modo a los que presentan sus tesis y a los que no. ¿Qué elemento diferencial se puede apreciar en aquellos que arriban a la meta? Se puede concluir que la contención institucional, la buena capacitación de aquellos que deben orientar y acompañar a los alumnos, como tutores, consejeros, directores además de la disposición personal del tesista.

Se puede concluir que para modificar la alta tasa de ingresantes, mediana tasa de egresados y baja tasa de titulados, es necesario políticas proactivas de carácter institucional que tiendan ajustar las demandas y exigencias junto con los recursos materiales

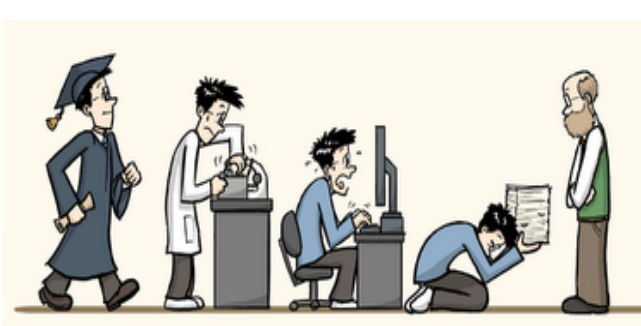

THE ORIGIN OF THE THESES y humanos que se requieren para llevar a buen término el proceso de producción de tesis. En muchas ocasiones no se trata de un problema de falta de recursos, sino de una adecuada administración de esos recursos. Hacer que los alumnos se titulen debe ser parte del proceso de formación. Suele observarse en la mayoría de las instituciones que hay una preocupación por dar contención a los alumnos en relación a que cuenten con cupos, docentes a cargo de materias, recursos bibliográficos y de las tics, apoyo económico a través de becas y subsidios, sin embargo, a la hora de finalizar las cursadas regulares y previo a la presentación de la tesis se advierte cierta orfandad en la que los tesistas quedan a la "deriva" para emprender una tarea que requiere un tiempo adecuado de aprendizaje. El seminario o taller de tesis muchas veces expone esta situación y trata de remediarla en la medida de la posible, pero no puede resolver hacia el pasado todo lo que no se hizo y hacia el futuro la suerte de acompañamiento que requieren los tesistas. En muchas ocasiones los directores de tesis pueden ser muy idóneos en su campo científico o profesional, pero se requiere más que eso para poder dirigir una investigación. No suele ser, en la mayoría de los casos, la extenuante y demandante tarea del director, cuando se encuentra llevada adelante adecuadamente, remunerada. Incluso muchas veces la buena voluntad de dirigir una tesis no se ve acompañada de la disponibilidad necesaria que ésta demanda.

Superar el cuello de botella de la elaboración y presentación de la tesis requiere de una toma de conciencia por parte de los tesistas de la tarea a realizar y de cómo deben ser ordenados los distintos pasos en el proceso de investigación a los efectos de no dilapidar esfuerzos y por parte de las instituciones la revisión en la implementación de recursos para la concreción de dicho proceso. No todo depende de la voluntad de los tesistas, ni de la contención institucional, pero ambos deben asistir a esa producción considerando el modo de generar prácticas y mecanismos qué, sin desmerecer la calidad de los resultados, contribuya a superar los obstáculos que se ponen en evidencia en todo momento en los claustros universitarios. 
118/ Perspectivas Metodológicas /19/Vol. II /Año 2017

\section{Bibliografía}

- Arnoux E: Escritura y producción de conocimientos en las carreras de postgrado (ed.)Buenos Aires, Santiago Arcos, 2009.

— Mombrú, A. Marjetic, A.: El hacedor de tesis, Ed. Pragma, Bs.As., 2002.

- Naishtat, F. y otros: Filosofía de la Universidad y conflicto de racionalidades. Colihue, Buenos Aires 2001.

- Wainerman,C.; Sautu, R.: La Trastienda de la Investigación, Bs.As., Ediciones Lumiere, 2001

\section{Bibliografía de la WEB}

— http://www.observatoriocts.org/files/.../formacion_de_postgrado. Formación de posgrado en América Latina - FINAL.pmd 28-10-2010

- http://repositorio.educacion.gov.ar:8080/dspace/bitstream/handle/123456789/109591 Anuario_de_Estadisticas_Universitarias_2010.pdf?sequen$\mathrm{ce}=1$

— http://www.coneau.gov.ar/CONEAU/index.php?option=com_content\&view=article\&id=92:normas-especificas-para-la-evaluacion-de-carreras-nuevas

- http://www.unrc.edu.ar/publicar/cde/05/Follari.htm

— http://www.comunicación y medios.com./Reflexión/Teorías /fin de siglo.htm. García Canclini: Comunicación fin de siglo. ¿Para dónde va nuestra investigación?

— http://www.scielo.org.ve/scieloOrg/php/Valarino, E. (2000/1997). Tesis a Tiempo. (2a. ed.). Barcelona, España: Grupo Editorial Carnero.

\section{Ilustraciones}

— Ilustración 1, p.2: http://www.escombrismo.blogspot.com.ar2012/03/01

— Ilustración 1, p.3: http://www.lunacreciente.blogspot.com.ar2006/12/01

- Ilustración 1, p. 6: http://www.doctorandos.com/agradecimientos-de-tesis $2012 / 14 / 12$

—Ilustración 1, p.11://http://www.phdcomics.com/comics/archive 2009/04/29 